\title{
$\beta$-Elemene Reverses Chemoresistance of Breast Cancer Cells by Reducing Resistance Transmission via Exosomes
}

\author{
Jun Zhang a,b,e He-da Zhang ${ }^{a, c}$ Yu-Feng Yao ${ }^{a}$ Shan-Liang Zhong ${ }^{d}$ Jian Hua Zhao ${ }^{d}$ \\ Jin Hai Tang ${ }^{a}$ \\ aDepartment of General Surgery, Nanjing Medical University Affiliated Cancer Hospital, Cancer Institute \\ of Jiangsu Province, Nanjing, ${ }^{b}$ Department of post Doctoral working station, Jinling Hospital Affiliated \\ to Medical College of Nanjing University, Nanjing, 'Graduate School, Xuzhou Medical College, Xuzhou, \\ dDepartment of Center of Clinical Laboratory Science,Nanjing Medical University Affiliated Cancer \\ Hospital, Cancer Institute of Jiangsu Province, Nanjing, ${ }^{e}$ Anhui University of Chinese Medicine, Hefei, \\ Anhui, China
}

\section{Key Word}

Breast cancer $\bullet$ Chemoresistance $\bullet \beta$-elemene $\cdot$ miRNA $\bullet$ Exosome

\begin{abstract}
Background: Currently, exosomes that act as mediators of intercellular communication are being researched extensively. Our previous studies confirmed that these exosomes contain microRNAs (miRNAs) that could alter chemo-susceptibility, which is partly attributed to the successful intercellular transfer of multidrug resistance (MDR)-specific miRNAs. We also confirmed that $\beta$-elemene could influence MDR-related miRNA expression and regulate the expression of the target genes PTEN and Pgp, which may lead to the reversal of the chemoresistant breast cancer (BCA) cells. We are the first to report these findings, and we propose the following logical hypothesis: $\beta$-elemene can mediate MDR-related miRNA expression in cells, thereby affecting the exosome contents, reducing chemoresistance transmission via exosomes, and reversing the drug resistance of breast cancer cells. Methods: MTT-cytotoxic, miRNA microarray, real-time quantitative PCR, Dual Luciferase Activity Assay, and Western blot analysis were performed to investigate the impact of $\beta$-elemene on the expression of chemoresistance specific miRNA and PTEN as well as Pgp in chemoresistant BCA exosomes. Results: Drug resistance can be reversed by $\beta$-elemene related to exosomes. There were 104 differentially expressed miRNAs in the exosomes of two chemoresistant BCA cells: adriacin (Adr) - resistant MCF-7 cells (MCF-7/Adr) and docetaxel (Doc) - resistant MCF-7 cells (MCF-7/Doc) that underwent treatment. Of these, 31 miRNAs were correlated with the constant changes in the MDR. The expression of miR-34a and miR-452 can lead to changes in the characteristics of two chemoresistant BCA exosomes: MCF-7/Adr exosomes (A/exo) and
\end{abstract}

J. Zhang and H.-d. Zhang contributed equally to this work.

Hai Tang

KARGER 125
Department of General Surgery, Nanjing Medical University Affiliated Cancer Hospital, Cancer Institute of Jiangsu, 42 Bai Zi Ting Road, Nanjing, Jiangsu,China. 210000 (P.R. China); E-Mail junzhang80122@sina.com, E-Mail jinhaitang11@hotmail.com 
MCF-7/Doc exosomes (D/exo). The PTEN expression affected by $\beta$-elemene was significantly increased, and the Pgp expression affected by $\beta$-elemene was significantly decreased in both cells and exosomes. $\beta$-elemene induced a significant increase in the apoptosis rate in both MCF-7/Doc and MCF-7/Adr cells. Conclusions: Drug resistance can be reversed by $\beta$-elemene, which can alter the expression of some MDR-related miRNAs, including PTEN and Pgp in MCF-7/Adr and MCF-7/Doc in cells. It can therefore affect the exosome contents and induce the reduction of resistance transmission via exosomes.

Copyright $(2015$ S. Karger AG, Basel

\section{Introduction}

One important reason for the failure of breast cancer chemotherapy is multi-drug resistance (MDR). However the underline mechanism has not been fully elucidated.

Adriamycin (Adr) and Docetaxel (Doc) are two of the most commonly used chemotherapy drugs in breast cancer. Elemene is a novel noncytotoxic anticancer drug, and previous studies have revealed that $\beta$-elemene might be an effective MDR reversing agent in cancer chemotherapy [1-4]; however, the underlying mechanism of $\beta$-elemene has not been fully elucidated.

Exosomes are small vesicles that are 50 to $100 \mathrm{~nm}$ in diameter. They are released upon the fusion of multivesicular bodies with plasma membranes from diverse cell types and act as mediators of intercellular communication, which are being increasingly researched. They contain miRNAs that can be transferred to target cells and induce epigenetic changes. Evidence suggests that miRNAs circulate in body fluids in a highly stable and cell-free form, which is likely due to their incorporation into exosomes [5-9]. Our previous study showed that the ability of MDR BCA cells in transmitting drug-resistance capacity is due to their release of exosomes. These exosomes could alter chemo-susceptibility in recipient sensitive cells, which partly contributes to the successful intercellular transfer of MDR-specific miRNAs and results in the reversal of the BCA chemoresistance [10]. We also confirmed that $\beta$-elemene could influence MDR-related miRNA expression and regulate the expression of the target genes PTEN and Pgp, which may lead to the reversal of the chemoresistant breast cancer cells [11]. However, no report has demonstrated the mechanisms of $\beta$-elemene in reversing MDR in BCA from exosomes. Therefore, we propose the following logical hypothesis: $\beta$-elemene can mediate MDR-related miRNA expression in cells by affecting the exosome contents, reducing chemoresistance transmission via exosomes to reverse the drug resistance of breast cancer and subsequently interrupting the development of drug-resistance in cancer cells and improving the treatment efficacy.

miRNAs target several mechanisms in drug-resistant breast cancer [12-16]. Previously, we showed that $\beta$-elemene significantly suppresses chemoresistant breast cancer cell growth and proliferation, which could influence MDR-related miRNA expression and regulate the expression of the target genes PTEN and Pgp [11]. In this study, we proved that $\beta$-elemene can mediate MDR-related miRNA and PTEN Pgp expression in exosomes, thus reducing chemoresistance transmission via exosomes to reverse the drug resistance in breast cancer, interrupting the development of drug resistance in cancer cells and improving the treatment efficacy (Fig. 8).

First, we confirmed that MCF-7/Adr and MCF-7/Doc could potentially spread chemoresistance to recipient cells and that exosomes could enhance this chemoresistance transmission. Second, the miRNA expression profiles show that drug resistant BCA cells and exosomes treated with $\beta$-elemene $(50 \mu \mathrm{M} / \mathrm{L}, 30 \mathrm{~h})$ may have characteristics that can significantly change the expression of miRNAs in the cells and exosomes. In addition, we confirmed that $\beta$-elemene induced a significant increase in the apoptosis rate in BCA cells, and we assessed PTEN and Pgp expression in exosomes that were treated with $\beta$-elemene. We concluded that $\beta$-elemene can mediate MDR-related miRNA (miR-34a, miR-452), Pgp, and the PTEN gene in cells and can therefore affect them in exosomes, thereby reducing chemoresistance transmission via exosomes and reversing drug resistance in breast cancer. In conclusion, our 


\section{Cellular Physiology Cell Physiol Biochem 2015;36:2274-2286 \begin{tabular}{l|l} 
Dor: 10.1159/000430191 & $\begin{array}{l}\text { O 2015 S. Karger AG, Basel } \\
\text { www.karger.com/cpb }\end{array}$ \\
\hline and Biochemistry & Published online: July 24, 2015
\end{tabular} \\ Zhang et al.: $\beta$-Elemene Reverses Chemoresistance of Breast Cancer via Exosomes}

results clearly indicate that $\beta$-elemene effectively sensitises drug resistant BCA cells to Doc and Adr through a signalling pathway that involves miRNA and gene regulation. This study could provide a better understanding of the roles that miRNAs and exosomes play in reversing drug resistance in BCA.

\section{Materials and Methods}

\section{Cell culture and CCK8-cytotoxic}

The human breast cancer cell line MCF-7 was purchased from ATCC (Rockville, MD, USA). The resistant sublines were successfully established from human breast cancer parental cell line MCF-7 by exposing MCF-7 to gradually increasing concentrations of Doc or Adr in vitro. Then, $10 \mu \mathrm{l}$ of CCK8 solution $(5 \mathrm{mg} / \mathrm{ml})$ was added to each well, and each experiment was performed in quadruplicate. The absorbance was measured at $570 \mathrm{~nm}$ using CliniBio128 (ASYS-Hitech, Austria)

\section{MiRNA microarray}

Total RNA, including miRNAs, was extracted using the MirVana miRNA Isolation Kit (Ambion, AM1560). The RNA was labelled using the FlashTag RNA Labelling Kit (Genishere), according to the manufacturer's recommendations. Hybridisation and washing were performed using the Affymetrix Fluidics Station 450 and Hybridisation Oven 640 under standard conditions. The Affymetrix GeneChip miRNA 2.0 Array contains 15,644 probe sets, including 1105 human mature miRNAs. The raw data were treated using the miRNA QC tool software (Affymetrix). Differentially expressed miRNAs were filtered to exclude all changes less than 2.0-fold compared with MCF-7/S.

\section{Dual Luciferase Activity Assay}

A recombinant lentiviral vector stable expression of green fluorescent protein was used in breast cancer cell lines. MCF-7/Doc and MCF-7 cells in the logarithmic growth phase were carried out in accordance with reagent instruction lentivirus infections. After 72 hours, the collected fluorescence was stronger after the addition of $2 \mu \mathrm{g} / \mathrm{ml}$ puromycin to the screen. After one week, $1 \mu \mathrm{g} / \mathrm{ml}$ puromycin was added, and three generations were cultured to observe the expression of the green fluorescence. Then, the green fluorescence MCF-7 cells, MCF-7/Adr cells and MCF-7/Doc cells were inoculated in equal amounts with $\beta$-elemene. Subsequently, they were treated with Doc and Adr for 24 hours. Luciferase activities were measured using a Dual Luciferase Reporter Assay System (Promega, USA) according to the manufacturer's instruction, and the renilla luciferase activity was normalised.

\section{Exosome isolation and identification}

Exosomes were harvested from supernatants of MCF-7/Adr, MCF-7/Doc and MCF-7 cultured in DMEM with 10\% exosome-depleted FBS by differential centrifugation and ultracentrifugation, as previously described. The exosomes were designated exo, A/exo, D/exo for simplicity. Briefly, cell culture media were sequentially centrifuged at $300 \mathrm{~g}$ for $10 \mathrm{mins}, 2,000 \mathrm{~g}$ for $15 \mathrm{mins}$, and 12,000 g for $30 \mathrm{mins}$ to remove floating cells and cellular debris. The filtrates were further ultracentrifuged by an Avanti J-30I (Beckman Coulter, USA) at $100,000 \mathrm{~g}$ for $2 \mathrm{hrs}$ at $4^{\circ} \mathrm{C}$ to collect exosomal pellets. Cytofluorimetric analysis (BD FACSCalibur, USA) was performed to detect surface molecules using a fluorescein isothiocyanate(FITC)-conjugated antibody directed against Tsg101 and calnexin (DakoCytomation, Denmark).

\section{Co-culture assays}

Our previous study showed that RNase abrogated the effects of A/exo and D/exo on an elevated G1/ G2 phase. The addition of exosomes with high concentrations of RNase inactivates the RNA. The significant reduction of exosome biological activities after RNase treatment suggests a putative transfer of RNA from A/exo and D/exo to recipient MCF-7/S [9]. A/exo, D/exo were treated with $5 \mathrm{U} / \mathrm{ml}$ RNase (Ambion, USA) for $3 \mathrm{~h}$ at $37 \mathrm{uC}$ (RNase A/exo, RNase D/exo), the reaction was stopped by addition of $10 \mathrm{U} / \mathrm{ml}$ RNase inhibitor (Ambion, USA) followed by ultracentrifugation. To investigate potential transmission of chemoresistance, either MCF-7/Adr, MCF-7/Doc or MCF- 7/S cells were mixed with GFP-S at equal proportions in 6-well plates. Following $72 \mathrm{hrs}$ of growth in co-culture, the cells were treated with $250 \mathrm{nM}$ adr and $50 \mathrm{nM}$ doc for 


\section{Cellular Physiology Cell Physiol Biochem 2015;36:2274-2286 \begin{tabular}{l|l} 
DOI: 10.1159/000430191 & $\begin{array}{l}\text { O 2015 S. Karger AG, Basel } \\
\text { www.karger.com/cpb }\end{array}$
\end{tabular}

$24 \mathrm{hrs}$. The percentage of GFP-S was measured by fluorescence-activated cell sorting (FACS). The number of residual GFP-S was equal to the residual cell number percentage of GFP-S. The Annexin-V-FITC binding on GFP-S was analysed by flow cytometry (BD FACSCalibur, USA) using FITC signal detector. After the cells had attached, the media was removed and fresh media containing MCF-7/Adr and MCF-7/Doc was added. Then, we added $20 \mu \mathrm{g} / \mathrm{ml} \mathrm{A} / \mathrm{exo}, \mathrm{D} / \mathrm{exo}$, RNase, $\beta$-elemene, $\mathrm{A} /$ exo $+\beta$-elemene, $\mathrm{D} / \mathrm{exo}+\beta$-elemene, $10 \mathrm{U} /$ ml RNase $+\beta$-elemene.

\section{Western blot}

Total protein was extracted and lysed in the RIPA buffer (Beyotime, Jiangsu, China). Equal amounts of proteins were separated by $10 \%$ SDS-PAGE and transferred to the polyvinylidene difluoride membranes (Sigma, Germany). The membranes were incubated with primary antibodies against human Pgp (1:100, Abcam, America), the horseradish peroxidase-conjugated secondary antibody (Kangwei Ltd, Beijing, China) was further incubated; the protein band was visualised by Chemiluminescence with pierce ECL kits (Millipore, Billerica, MA).

\section{Statistical analysis}

All experiments were performed in triplicate, and representative data were shown from three separate experiments. A statistical analysis was performed using a t-test or one-way ANOVA and the Spearman rank test using the SPSS 16.0 statistical software. All experiments were performed in triplicate, and $\mathrm{p}<0.05$ was considered statistically significant.

\section{Results}

Characterisation of exosomes

To investigate their correlation with drug intervention, we first collected A/exo, D/exo from supernatants of MCF-7/Adr, MCF-7/Doc cells through a series of centrifugation and ultracentrifugation steps. The characteristics of exosomes were determined by electron microscopy, Western blot analysis and flow cytometry. Western blot analysis for the exosome related proteins Tsg101 and $\beta$-actin and endoplasmic reticulum protein calnexin revealed that the exosomes were positive for Tsg101, calnexin and $\beta$-actin (Fig. 1A). Moreover, according to the FACS analysis, all exosomes expressed surface molecule CD63 (Fig. 1B).

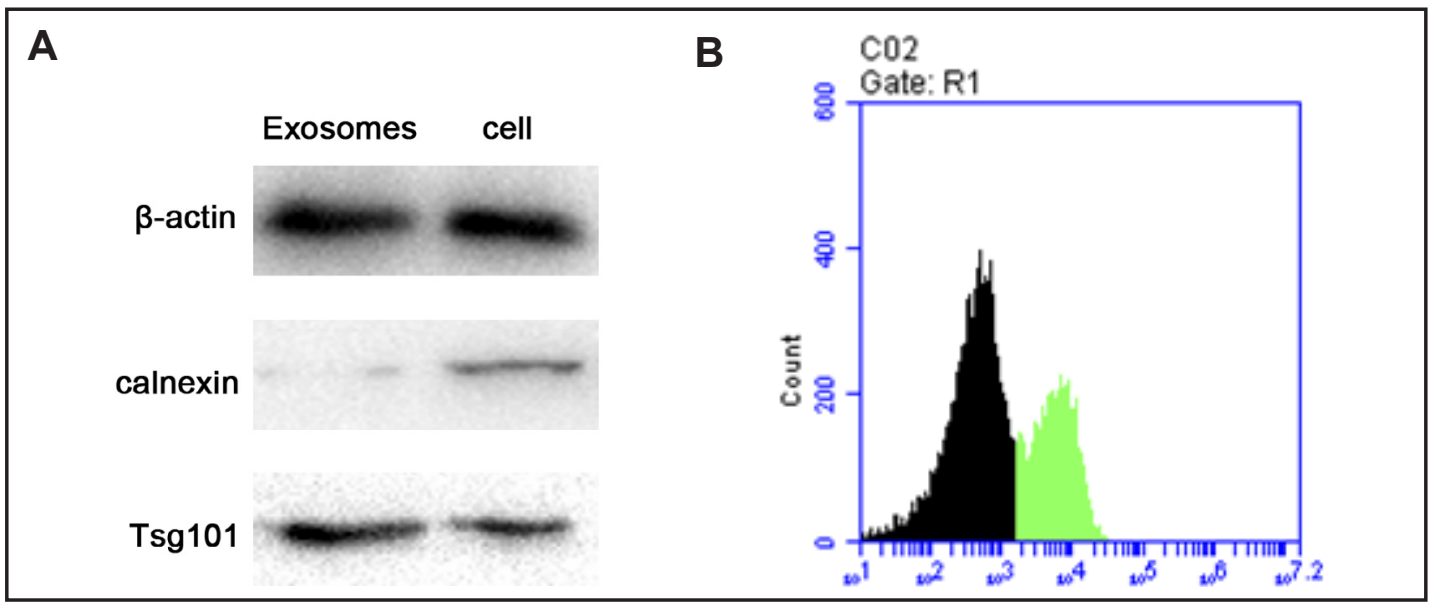

Fig. 1. Characterisation of exosomes (A): Western blot for the exosome-related proteins Tsg101 and b-actin and endoplasmic reticulum protein calnexin in D/exo and MCF-7/Doc. D/exo is positive for Tsg101 and b-actin, but no calnexin is detected. (B): Representative cytofluorimetric analysis of D/exo that shows the expression of CD63 (green) surface molecule. Black represents the isotypic control. For A and B, exosome preparations of A/exo, D/exo and S/exo were analysed, with similar results. 


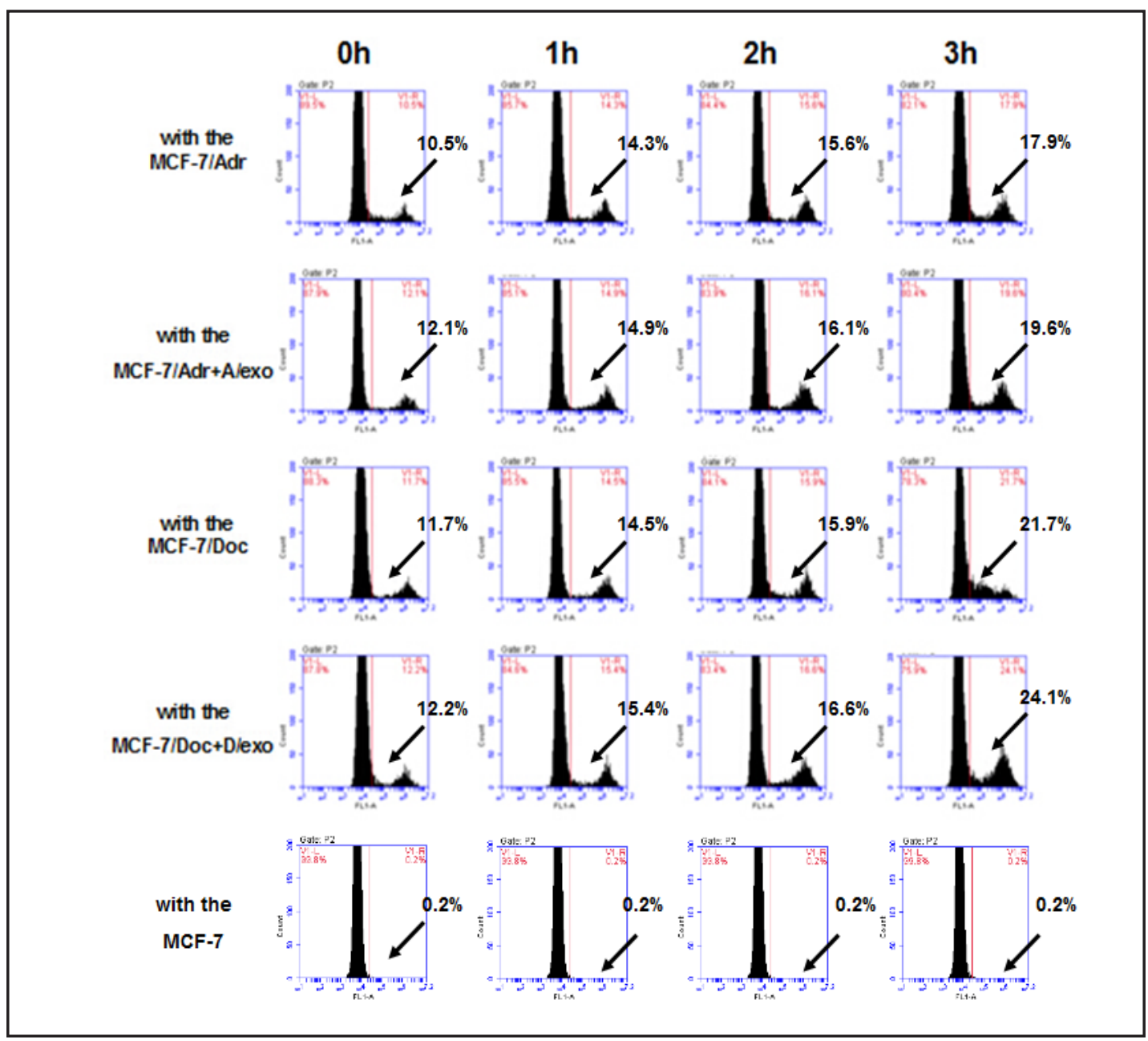

Fig. 2. Co-culture of GFP-S cells and MCF-7/Adr or MCF-7/Doc resulted in more and more surviving GFP-S (\%) after drug exposure with the change of time $(0 \mathrm{~h}, 1 \mathrm{~h}, 2 \mathrm{~h}, 3 \mathrm{~h})$, and the GFP-S of the two group added exosomes was significantly more. (1)MCF-7/Adr vs. MCF-7/Adr +A/exo:1h:14.9\%, 14.3\%; 2h:16.1\%, 15.6\%; 3h:19.6\%, 17.9\% (2)MCF-7/Doc vs. MCF-7/Doc +D/exo: 1h: 15.4\%, 14.5\%; 2h:16.6\%, 15.9\%; 3h:24.1\%, $21.7 \%$.

Exosomes can enhance chemoresistance transmission in co-cultures

In our previous study, exosome-transmitted chemoresistance was subsequently assessed in exosome-treated MCF-7/S by analysing the cell cycle distribution and determining the apoptotic rates [10]. Here, we aimed to verify this finding again by transfecting MCF7/S before seeding it with the lentivirus encoding green fluorescent protein (called GFP-S). GFP-S and daughter GFP-S with inherited green fluorescence could be clearly identified in co-cultures over several days. Then, they were maintained in a 1:1 ratio for $72 \mathrm{hrs}$ under the following conditions: i) with the MCF-7/Adr, ii) with the MCF-7/Adr $+20 \mu \mathrm{g} / \mathrm{ml} \mathrm{A} /$ exo, iii) with the MCF-7/Doc, iv) with the MCF-7/Doc $+20 \mu \mathrm{g} / \mathrm{ml}$ D/exo, and V) with the MCF-7/S, after which the resistance status was assessed in the presence of $250 \mathrm{nM}$ adr and $50 \mathrm{nM}$ doc at different time points $(0 \mathrm{~h}, 1 \mathrm{~h}, 2 \mathrm{~h}, 3 \mathrm{~h})$. Co-culturing GFP-S cells with MCF-7/Adr or MCF-7/Doc increased in the amount of surviving GFP-S after drug exposure compared with MCF-7 cells, even with the change in duration, and significantly more GFP-S in both groups added exosomes. These results suggested that MCF-7/Adr and MCF-7/Doc could potentially spread chemoresistance to recipient cells in a time-dependent manner and that exosomes can enhance this transmission of resistance (Fig. 2). 
Fig. 3. (A) and (B): The green fluorescent cells expression by dual luciferase activity assay in MCF7/Doc and MCF-7/ Adr cells, MCF-7/ Doc and MCF-7/Adr cells with $\beta$-elemene, MCF-7/Doc and MCF$7 / \mathrm{Adr}$ cells with $\beta$ - elemene + exo (MCF-7), MCF-7/ Doc and MCF-7/ Adr cells with $\beta$-elemene + RNase ( $\beta$-elemene: $50 \mu \mathrm{M} / \mathrm{L}$ for $30 \mathrm{~h}$, exo: $20 \mu \mathrm{g}$ / $\mathrm{ml}$, RNase, $5 \mathrm{U} / \mathrm{ml}$ ). (C) and (D): Apoptotic rate of GFP-S was determined after cell mixture was treated with $50 \mathrm{~nm}$ Doc or $250 \mathrm{~nm}$ Adr for 30 h. ${ }^{* *} \mathrm{P}<0.01$, MCF-7/ MCF-7/Adr or MCF$7 / \mathrm{Doc}+\beta$-ele; MCF$7 / \mathrm{Adr}$ or MCF-7/Doc $+\beta$-ele vs. MCF-7/Adr or MCF-7/Doc $+\beta$-ele +exo. Adr or MCF-7/Doc vs.

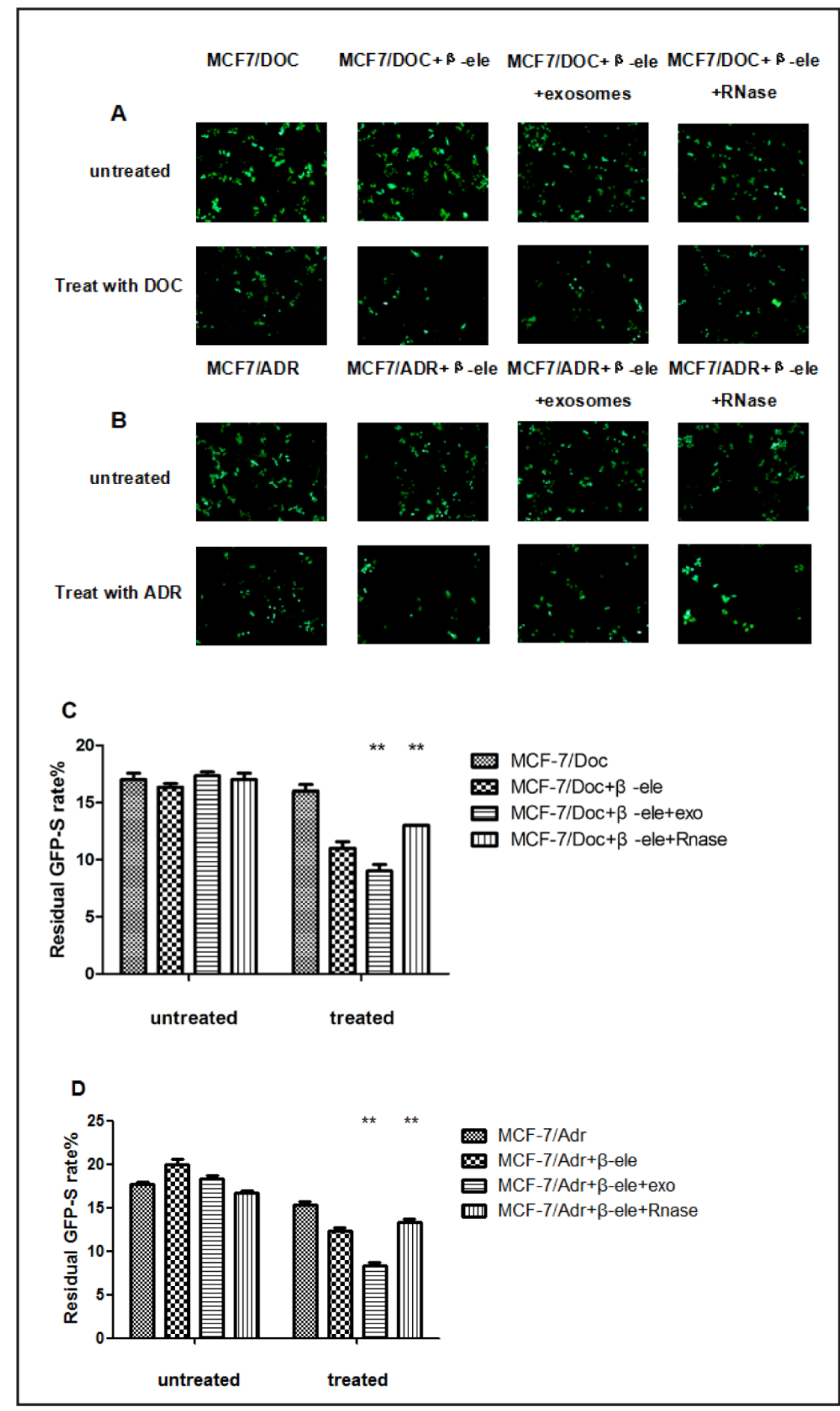

$B$-elemene can reverse chemoresistance related to exosomes

We confirmed that MCF-7/Adr and MCF-7/Doc could potentially spread chemoresistance to recipient cells, which is likely due to the exosomes shed by drug-resistance cells. Here, we confirmed the important role of exosomes in $\beta$-elemene reversing drug resistance. After $\beta$-elemene treatment ( $50 \mu \mathrm{M} / \mathrm{L}$ ) for $30 \mathrm{hrs}$, by Doc and Adr, the remnants from green fluorescent cells were significantly different between MCF-7/Doc, MCF-7/Adr, MCF-7/Doc + D/exo, MCF-7/Adr + A/exo, MCF-7/Doc +Rnase, MCF-7/Adr + Rnase (Fig. 3AB). After MCF7/Doc and MCF-7/Adr cells had been treated with $\beta$-elemene and were exposed to the drug, 


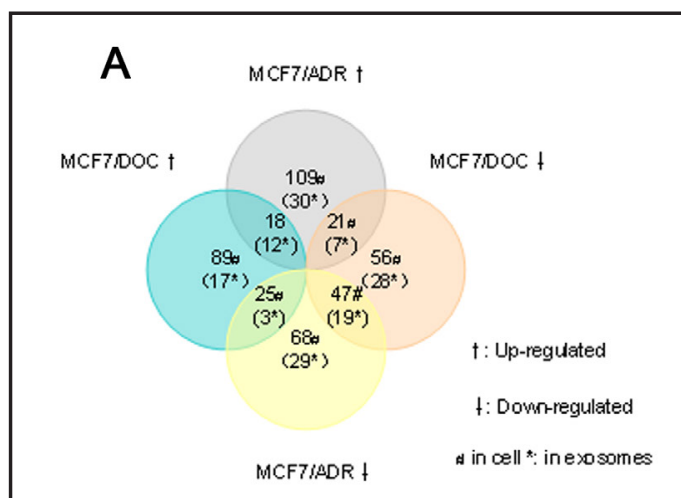

Fig. 4. (A) and (B): miRNA microarray compared with the miRNA profile of MCF-7/Doc and MCF$7 /$ Adr cells and exosomes that were previously tested, which shows the differentially expressed miRNAs in MCF-7/Doc and MCF-7/Adr cells and exosomes with $\beta$-elemene intervention $(80 \mu \mathrm{M} / \mathrm{L})$ for 24 h. (C): Hierarchical cluster analysis of miR$\mathrm{NA}$ expression profiles in A/exo and D/exo with $\beta$-elemene intervention compared with $\mathrm{A} / \mathrm{exo}$ and D/exo without intervention. Black indicates a median transcript level, red indicates a transcript level above the median level, and green represents a transcript level below the median level of the particular assay, as measured in all samples.

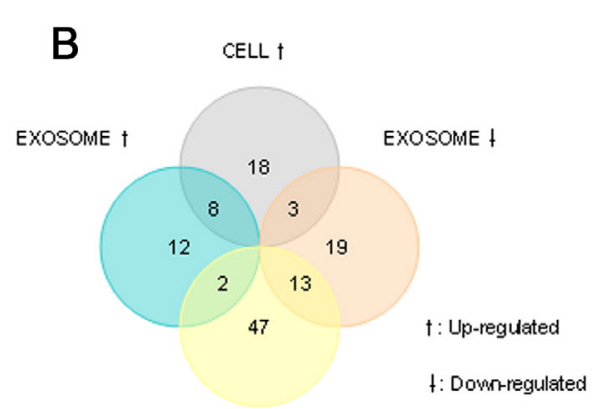

CELL +

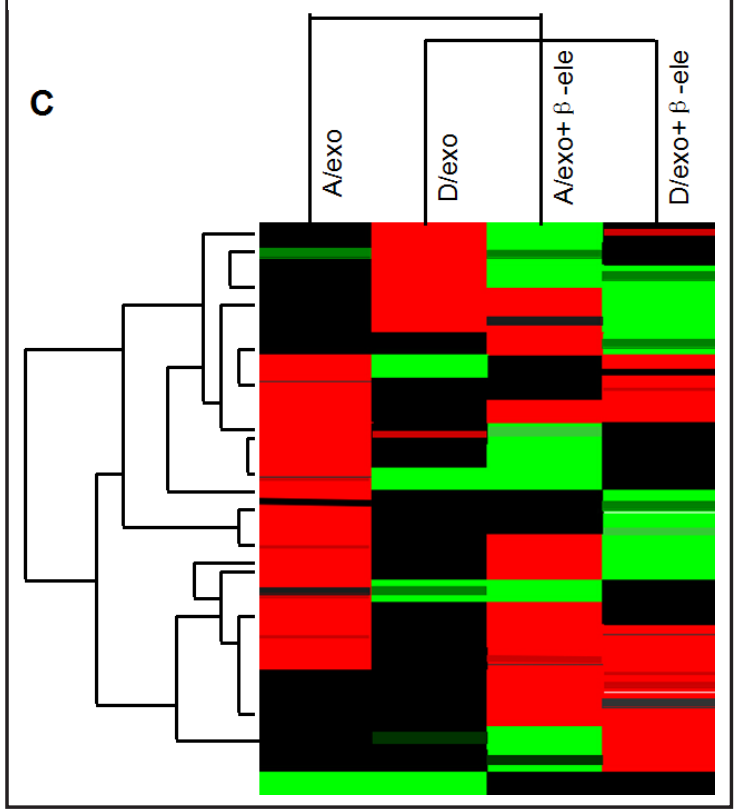

the residual green fluorescent cells was significantly reduced compared with untreated MCF$7 /$ Doc and MCF-7/Adr, which indicated that drug resistance can be reversed by $\beta$-elemene. After the MCF-7/Doc+D/exo and MCF-7/Adr+A/exo had been treated with $\beta$-elemene intervention and were exposed to the drug, the residual green fluorescent cells were significantly reduced compared with MCF-7/Doc and MCF-7/Adr with $\beta$-elemene treatment only. Compared with the MCF-7/Doc and MCF-7/Adr with $\beta$-elemene treatment only and MCF-7/ Doc $+\mathrm{D} /$ exo and MCF-7/Adr $+\mathrm{A} /$ exo with $\beta$-elemene treatment, the residual green fluorescent cells of MCF-7/Doc, MCF-7/Adr + Rnase with $\beta$-elemene treatment were significantly increased, which suggested that drug resistance can be reversed by $\beta$-elemene related to exosomes (Fig. 3CD: Supporting for 3AB).

Expression profile ofmiRNAs in the exosomes of MCF-7/Doc and MCF-7/Adr with $\beta$-elemene intervention

We recently analysed the miRNA profiles of MCF-7, MCF-7/Adr, ,MCF-7/Doc cells and exosomes, as well as MCF-7/Adr and MCF-7/Doc cells with $\beta$-elemene treatment by microarray $[10,11]$. To test the hypothesis that $\beta$-elemene could mediate the MDR-specific miRNAs via exosomes, we tested the miRNA profiles of MCF-7/Adr and MCF-7/Doc exosomes after $\beta$-elemene treatment.

Combined with our previous research, this study showed that there were 322 differentially expressed miRNAs among the 1,200 miRNAs (at least 2.0-fold changes) in the two cells with intervention. Compared with MCF-7/Adr and MCF-7/Doc cells, 18 miRNAs were up-regulated, and 47 miRNAs were down-regulated in both cells. Compared with A/exo and 
Fig. 5. The two miRNAs (miR34a, miR452) with consistent expression changes in D/exo and A/exo after $\beta$-elemene intervention $(50 \mu \mathrm{M} / \mathrm{L})$ for $30 \mathrm{~h}$. ${ }^{* *} \mathrm{P}<0.01, \mathrm{~A} /$ exo or $\mathrm{D} /$ exo vs. A/ exo $+\beta$-ele or $\mathrm{D} /$ exo $+\beta$-ele.

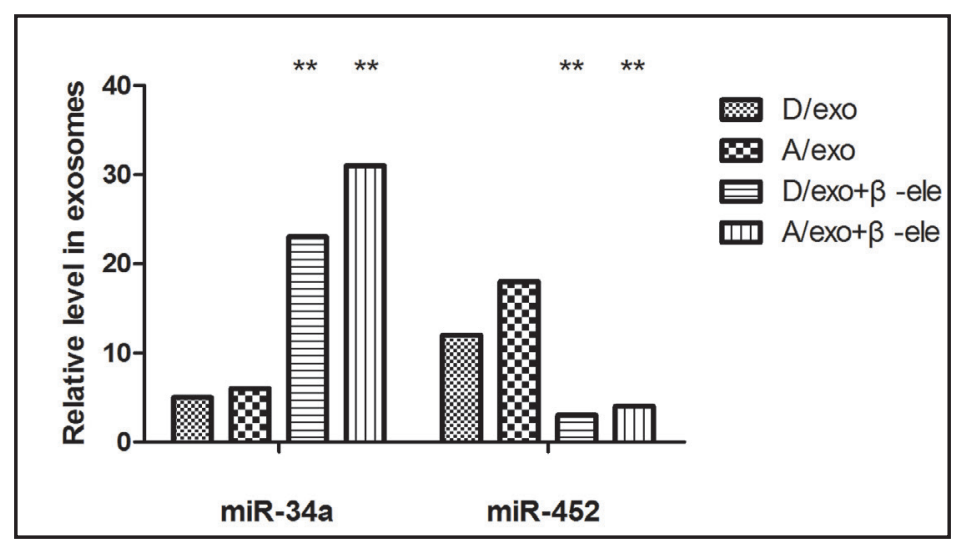

Table. 1. Significantly changed miRNAs in MCF-7/Doc and MCF-7/Adr cells and exosomes with $\beta$-elemene intervention $(50 \mu \mathrm{M} / \mathrm{L})$ for $30 \mathrm{~h}$ compared with the expression of miRNAs in MCF-7/Doc and MCF-7/Adr cells and exosomes that were previously tested and confirmed targets or pathway. (Criteria differences for Ratio > 2.5)

\begin{tabular}{|c|c|c|c|c|c|}
\hline $\begin{array}{l}\text { Significantly } \\
\text { regulation } \\
\text { (in two cells) }\end{array}$ & FC & $\begin{array}{l}\text { Confirmed targets or } \\
\text { pathway }\end{array}$ & $\begin{array}{l}\text { Significantly } \\
\text { regulation } \\
\text { (in two exosom }\end{array}$ & $\mathrm{FC}$ & $\begin{array}{l}\text { Confirmed targets or } \\
\text { pathway }\end{array}$ \\
\hline hsa-miR-320d $\uparrow$ & 2.56 & & hsa-miR-501 $\uparrow$ & 2.91 & HBXYP \\
\hline hsa-miR-34a $\uparrow$ & 6.83 & NOTCH1 BIRC3 & hsa-miR-34a $\uparrow$ & 5.21 & NOTCH1 BIRC3 \\
\hline hsa-miR-4284 $\uparrow$ & 2.79 & & hsa-miR-452 $\uparrow$ & 4.72 & TCF4,LEF 1,Bmi-1 \\
\hline hsa-miR-500 $\uparrow$ & 3.05 & & hsa-miR-139 † & 3.67 & HER2 Rho-kinace2 \\
\hline hsa-miR-193b $\uparrow$ & 2.99 & & hsa-miR-193b & 2.81 & PTEN/P13K Mcll \\
\hline hsa-miR-200c $\uparrow$ & 3.56 & P27/Kip1 PTEN/Akt & hsa-miR-200c $\downarrow$ & 3.52 & P27/Kip1 PTEN/Akt \\
\hline hsa-miR-155 । & 2.75 & FOXO3 RHOA PTEN & hsa-miR-152 & 2.65 & DNMT1 ADAM17Smads \\
\hline hsa-miR-145 । & 2.51 & ESR1 IGFR IRS1 & hsa-miR-3138 & 3.79 & \\
\hline hsa-miR-424 & 3.24 & & hsa-miR-452 & 5.32 & TCF4,LEF1,Bmi-1 \\
\hline hsa-miR-455 & 3.68 & & hsa-miR-877 & 4.23 & \\
\hline hsa-miR-503 & 2.88 & & hsa-miR-1908 & 2.89 & FOXO3 RHOA PTEN \\
\hline hsa-miR-29a $\downarrow$ & 8.32 & PTEN & & & \\
\hline hsa-miR-125b d & 5.77 & $\mathrm{Bcl}-2$ & & & \\
\hline hsa-miR-222 & 2.63 & PTEN P27 P57 BMF & & & \\
\hline hsa-miR-767 & 3.39 & & & & \\
\hline hsa-miR-152 & 2.67 & DNMTISmads & & & \\
\hline hsa-miR-452 & 7.91 & TCF4,LEF1,Bmi-1 & & & \\
\hline \multirow[t]{2}{*}{ hsa-miR-181a } & 6.63 & BCRP/ABCG2ATM & & & \\
\hline & & Bc12 PCAF TRMP3 & & & \\
\hline hsa-miR-21 & & PTEN Bel-2 TPM1 & & & \\
\hline
\end{tabular}

D/exo, there were 104 differentially expressed miRNAs in the two exosomes with intervention. Among the 104 miRNAs, 31 miRNAs were correlated with the constant changes of the MDR in two exosomes: 12 miRNAs were up-regulated and 19 were down-regulated in both exosomes; 17 miRNAs were up-regulated and 28 were down-regulated in D/exo only; 30 miRNAs were up-regulated and 29 were down-regulated in A/exo only; 3 miRNAs were upregulated in $\mathrm{D} / \mathrm{exo}$ but down-regulated in $\mathrm{A} / \mathrm{exo}$; and 7 miRNAs were down-regulated in $\mathrm{D} /$ exo but up-regulated in A/exo (Fig. 4A, Fig. 4C).

Among the miRNAs, 21 miRNAs were correlated with the constant changes in the MDR in the two cells and exosomes: 8 miRNAs were up-regulated and 13 were down-regulated in two cells and exosomes; and 3 miRNAs were up-regulated in two cells and down-regulated in two exosomes. In addition, 2 miRNAs were up-regulated in both exosomes and downregulated in both cells (Fig. 4B).

Among the 322 miRNAs, 6 miRNAs were significantly up-regulated, and 12 miRNAs were significantly down-regulated in both cells. Among the 104 miRNAs, 4 miRNAs were significantly up-regulated, and 7 were significantly down-regulated in both exosomes (criteria differences for Ratio > 2.5) (Table 1). Among these miRNAs, some were related to exosomes and drug resistance. 
Fig. 6. (A): $\beta$-elemene induced a significantly increased apoptosis rate in MCF-7/Doc and MCF-7/ Adr cells (B): The apoptosis rate in MCF-7/Doc cells was as follows: control, $1.90 \% \pm 0.52 \%$ vs. MCF-7/ Doc $+\beta$-ele cells, $20.61 \% \pm 1.03 \%$ $(\mathrm{p}<0.05)$, mean \pm SD. Similarly, the apoptosis rate in MCF-7/ Adr cells was as follows: control, $1.89 \% \pm 0.75 \%$ vs. MCF-7/Adr + $\beta$-ele, $19.23 \% \pm 1.92 \% \quad(\mathrm{p}<0.05)$, mean \pm SD.

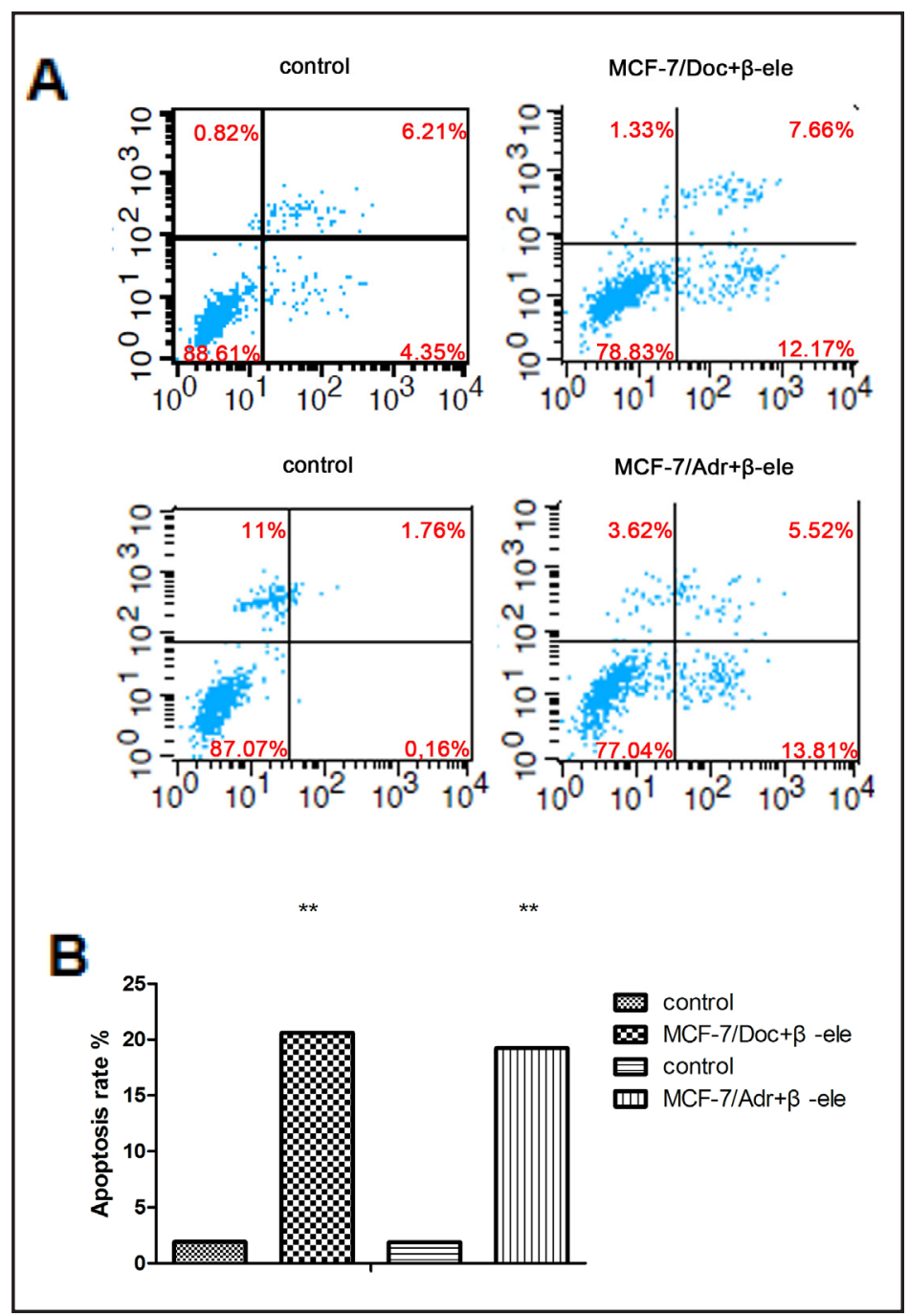

$\beta$-elemene reverses breast cancer cell resistance by mediating related miRNA in exosomes

In our previous study, four drug-specific miRNAs demonstrated significant reversal changes (miR-34a $\uparrow$, miR-222 $\downarrow$, miR-452 $\downarrow$ and miR-29a $\downarrow$ )[16]. In this study, two of these miR-

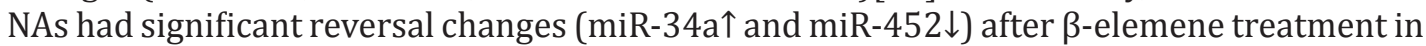
D/exo and A/exo compared with D/exo and A/exo without intervention (Fig. 5). This finding suggests that $\beta$-elemene may modulate the MDR-specific miRNA to reverse breast cancer cell chemoresistance, which is partly due to exosomes.

\section{$\beta$-elemene induces apoptotic cell}

$\beta$-elemene resulted in a significantly increased apoptosis rates in MCF-7/Doc cell and MCF-7/Adr cells compared with negative controls (Fig. 6A). The apoptosis rate in MCF-7/ Doc cells was as follows: control, $1.90 \% \pm 0.52 \%$ vs. MCF-7/Doc $+\beta$-ele cells, $20.61 \% \pm 1.03 \%$ $(\mathrm{p}<0.05)$, mean \pm SD. Similarly, the apoptosis rate in MCF-7/Adr and MCF-7/Doc cells was as follows: control, $1.89 \% \pm 0.75 \%$ vs. MCF-7 /Adr $+\beta$-ele, $19.23 \% \pm 1.92 \%(\mathrm{p}<0.05)$, mean \pm SD. (Fig. 6B).

$\beta$-elemene treatment alters the expression of PTEN and Pgp protein in breast cancer exosomes

We have previously performed Western blot analysis of P-gp expressions in MCF-7/S, MCF-7/DOC, and corresponding exosomes. The results show that MCF-7/DOC expressed 


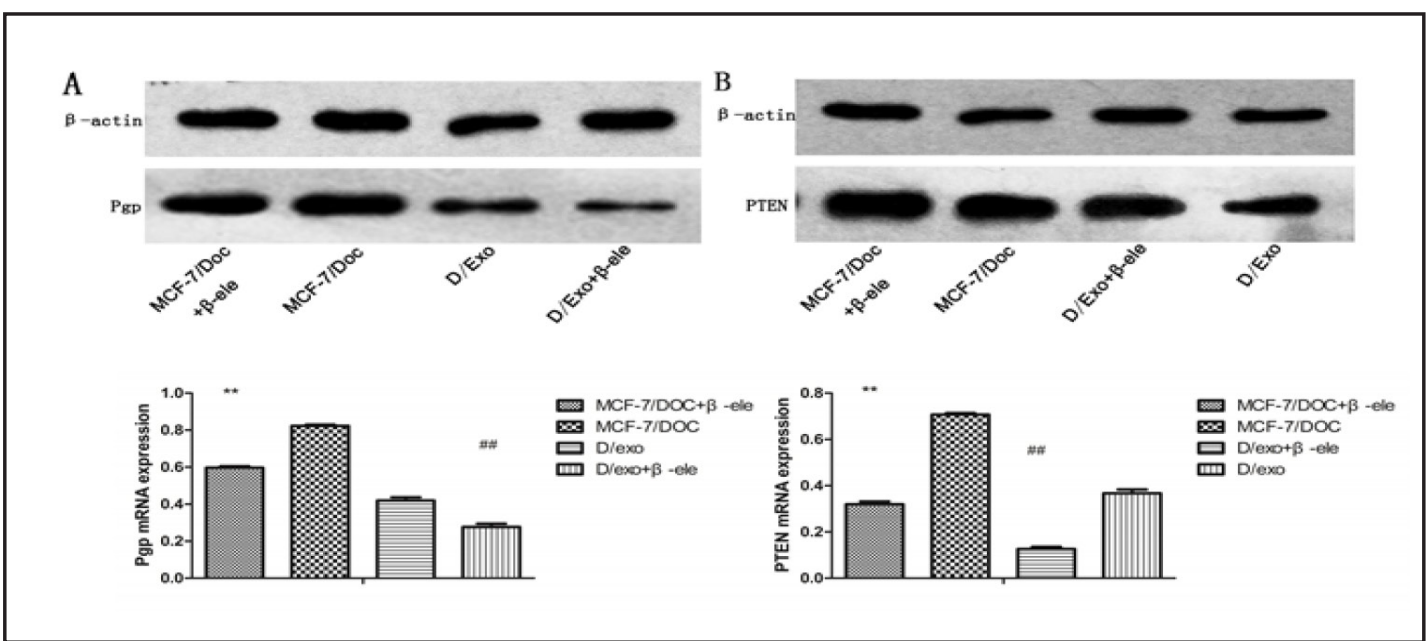

Fig. 7. (A): The expression of Pgp mRNA, Pgp protein levels in MCF-7/DOC cells and exosomes with $\beta$-elemene intervention ( $50 \mu \mathrm{M} / \mathrm{L})$ by western blot. (B): The expression of PTEN mRNA, PTEN protein levels in MCF-7/DOC cells and exosomes with intervention **\#\#P<0.01, MCF-7/Doc vs. MCF-7/Doc + $\beta$-ele; D/exo vs. D/exo + $\beta$-ele.

higher P-gp expression levels than MCF-7/S. The P-gp expression pattern of exosomes was similar to that of the original cells [17]. In addition, we confirmed that $\beta$-elemene could regulate the expression of the target genes PTEN and Pgp [11]. We utilised Western blot analysis to determine whether the potential expression of PTEN and Pgp changes in exosomes. The results revealed that the PTEN expression in MCF-7/Doc cells was significantly increased when they were treated with $\beta$-elemene and that its expression increased in MCF-7/Doc exosomes compared with untreated exosomes (Fig. 7A,B). The expression of Pgp was decreased in both MCF-7/Doc cells and exosomes treated with $\beta$-elemene compared with untreated exosomes (Fig. 7A,B).

\section{Discussion}

Breast cancer is the most common cancer in women worldwide. Previously, we showed that $\beta$-elemene significantly suppresses breast cancer cell growth and proliferation, which could influence MDR-related miRNA expression and regulate the expression of the target genes PTEN and Pgp [11]. In this study, we proved that $\beta$-elemene could mediate MDR-related miRNA expression in exosomes, which can then regulate the corresponding target genes through the gene regulatory network to interrupt the development of drug-resistance in cancer cells. This, in turn, would improve the treatment efficacy, which is likely partly due to the reduction of chemoresistance transmission via exosomes (Fig. 8).

Our previous study showed that P-gp expression patterns of exosomes were similar to those of the originated cells. The P-gp expression of MCF-7/S increased after incubation with DOC/exo and was affected by the amount of exosomes, which are effective in transferring both drug resistance and P-gp from drug-resistant breast cancer cells to sensitive ones. The delivery of P-gp via exosomes may be one of the mechanisms responsible for exosome-mediated drug resistance transfer. Studies have shown that PTEN secretion in exosomes has been demonstrated by the following independent groups: Ndfip1 is required for exporting PTEN in exosomes; miR-34a is associated with drug resistance in cancer; and increased miR-34a expression was observed in the present study in MCF-7/ADR and MCF-7/DOC chemoresistance breast cancer cells. Studies have shown that the dysregulation of miR-452 plays an important role in the acquired adriamycin-resistance of breast cancer by targeting insulin-like growth factor-1 receptor (IGF-1R). MDR of tumour cells is often associated with the overex- 
Fig. 8. Schematic diagram of chemoresistance transmission and the reduction of resistance transmission via exosomes (A): Drug-resistant tumour tissues can potentially spread chemoresistance to recipient-sensitive cells; $\beta$-elemene stopped and reduced this transmission (B): Drug-resistant cells spread miRNA to recipient sensitive cells via exosomes, thereby regulating target protein and MDR-related miRNA to induce chemoresistance in sensitive cells, whereas $\beta$-elemene stops and regulates this (C): $\beta$-elemene reverses the chemoresistance of breast cancer cells by regulating chemoresistance transmission by microRNA and exosomes.

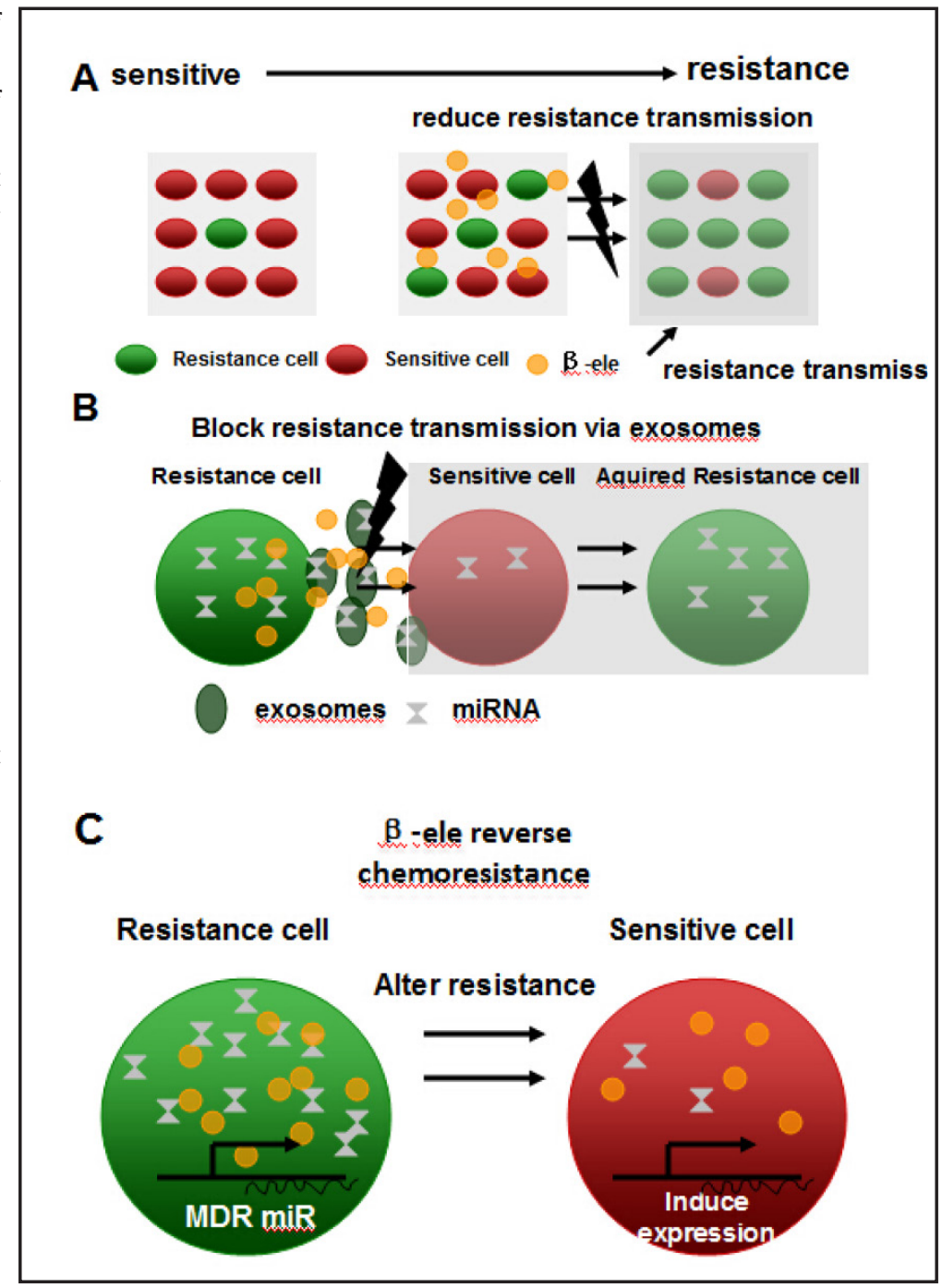

pression of Pgp and a lower expression of PTEN, which leads to chemotherapeutic failure and is often associated with some miRNA [17-25].

First, we confirmed that MCF-7/Adr and MCF-7/Doc could potentially spread chemoresistance to recipient cells and that exosomes could enhance this chemoresistance transmission. Second, the miRNA expression profiles shows that drug resistant BCA cells and exosomes with $\beta$-elemene intervention $(50 \mu \mathrm{M} / \mathrm{L}, 30 \mathrm{~h})$ may have characteristics that can significantly change the expression of miRNAs. In addition, we confirmed that $\beta$-elemene resulted in a significantly increased apoptosis rate in BCA cells, and we assessed PTEN and Pgp expression in breast cancer exosomes treated with $\beta$-elemene.

We conclude that $\beta$-elemene can mediate MDR-related miRNA (miR-34a and miR-452), Pgp and PTEN gene in exosomes to reverse the drug resistance of breast cancer. Our results clearly indicate that $\beta$-elemene effectively sensitised drug resistant BCA cells to Doc and Adr through a signalling pathway involving regulation of miRNA and genes. Exosomes contain miRNAs and some proteins that can be transferred to target cells; therefore, $\beta$-elemene can alter the expression of some MDR-related miRNAs, PTEN and Pgp in MCF-7/Adr and MCF-7/ Doc cells, affect the exosome contents and induce the reduction of resistance transmission via exosomes.

In summary, we were able to show that $\beta$-elemene causes a strong anticancer effect and induces BCA cell apoptosis. $\beta$-elemene can effect miRNA expression in cells and exosomes, reverse drug resistance and partly reduce resistance transmission via exosomes. This study 


\section{Cellular Physiology Cell Physiol Biochem 2015;36:2274-2286 \begin{tabular}{l|l} 
Dol: 10.1159/000430191 & $\begin{array}{l}\text { 2015 s. Karger AG, Basel } \\
\text { www.karger.com/cpb }\end{array}$ \\
\hline
\end{tabular} \\ Zhang et al.: $\beta$-Elemene Reverses Chemoresistance of Breast Cancer via Exosomes}

could contribute to a better understanding of the important roles of miRNAs and exosomes in reversing drug resistance in BCA. Moreover, this study provides a novel insight into the molecular mechanisms by which $\beta$-elemene reverses tumour resistance by using the latest technology to detect miRNA and exosome communication mechanisms. The strategic indepth exploration of MDR reducing expression mechanisms could help establish an alternative way of improving chemotherapy treatments.

\section{Acknowledgements}

This work was supported by grants from the National Natural Science Foundation of China (81272470), the Natural Science Foundation of Jiangsu Province (20131016) and Natural Science Foundation of Anhui Province (1408085QH184).

\section{Disclosure Statement}

The authors declare no conflicts of interest.

\section{References}

1 Zhang B, Zhang K, Liu Z, Hao F, Wang M, Li X, Yin Z, Liang H: Secreted Clusterin gene silencing enhances chemosensitivity of a549 cells to cisplatin through AKT and ERK1/2pathways in vitro. Cell Physiol Biochem 2014;33:1162-1175.

2 Zhang X, Zhang Y, Li Y: $\beta$-elemene decreases cell invasion by upregulating E-cadherin expression in MCF-7 human breast cancer cells. Oncol Rep 2013;30:745-750.

3 Xu HB, Li L, Fu J: Reversion of multidrug resistance in a chemoresistant human breast cancer cell line by $\beta$-elemene. Pharmacology 2012; 89: 303-312.

4 Guo HQ Zhang GN, Wang YJ: $\beta$-elemene, a compound derived from Rhizoma zedoariae, reverses multidrug resistance mediated by the ABCB1 transporter. Oncol Rep 2014;31:858-866.

5 Azmi AS, Bao B, Sarkar FH: Exosomes in cancer development, metastasis, and drug resistance: a comprehensive review. Cancer Metastasis Rev 2013;32:623-642.

6 Kahlert C, Kalluri R: Exosomes in tumor microenvironment influence cancer progression and metastasis. J Mol Med (Berl) 2013;91:431-437.

7 Hannafon BN, Ding WQ: Intercellular Communication by Exosome-Derived microRNAs in Cancer. Int J Mol Sci 2013;14:14240-14269.

8 Morel L, Regan M, Higashimori H, Ng SK, Esau C: Neuronal exosomal miRNA-dependent translational regulation of astroglial glutamate transporter GLT1. J Biol Chem 2013;288:7105-7116.

9 Chen X, Liang H, Zhang J, Zen K, Zhang CY: Secreted microRNAs: a new form of intercellular communication. Trends Cell Biol 2012;22:125-132.

10 Chen X, Liu X, Lv M, Chen L, Zhao H, Tang J: Exosomes from Drug-Resistant Breast Cancer Cells Transmit Chemoresistance by a Horizontal Transfer of MicroRNAs. PLOS One 2014;9: e95240

11 Zhang J, Zhang He, Chen L, Sun D, Tang J: $\beta$-Elemene Reverses Chemoresistance of Breast Cancer via Regulating MDR-Related MicroRNA Expression. Cell Physiol Biochem2014,34:2027-2037

12 Pan W, Wang H, Jian R, Ye Z: MicroRNA-27a promotes proliferation, migration and invasion by targeting MAP2K4 in human osteosarcoma cells. Cell Physiol Biochem 2014;33:402-412.

$13 \mathrm{Ku}$ KR, Yu OV, Bel FA: MicroRNA-mediated drug resistance in breast cancer. Clin Epigenetics 2011;2:171185.

14 Jiao X1, Zhao L, Ma M, BaiX, WeiM: MiR-181a enhances drug sensitivity in mitoxantone-resistant breast cancer cells by targeting breast cancer resistance protein (BCRP/ABCG2). Breast Cancer Res Treat 2013;139:717-730. 


\section{Cellular Physiology Cell Physiol Biochem 2015;36:2274-2286

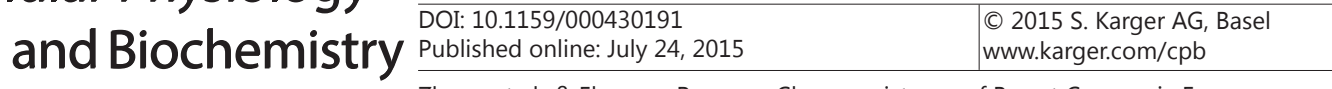 \\ Zhang et al.: $\beta$-Elemene Reverses Chemoresistance of Breast Cancer via Exosomes}

15 Li XJ, Ji MH, Zhong SL, Zha QB, Xu JJ, Zhao JH, Tang JH: MicroRNA-34a modulates chemosensitivity of breast cancer cells to adriamycin by targeting Notch1. Arch Med Res 2012;43:514-521.

16 Zhong S, Li W, Chen Z, Xu J, Zhao J: MiR-222 and miR-29a contribute to the drug-resistance of breast cancer cells. Gene 2013;531:8-14.

17 Lv MM, Zhu XY, Chen WX, Zhong SL, Hu Q, Ma TF, Zhang J, Chen L, Tang JH, Zhao JH: Exosomes mediate drug resistance transfer in MCF-7 breast cancer cells and a probable mechanism is delivery of P-glycoprotein. Tumour Biol 2014;35:10773-10779.

18 Gabriel K, Ingram A, Austin R, Kapoor A, Tang D, Majeed F, Qureshi T, Al-Nedawi K: Regulation of the tumor suppressor PTEN through exosomes: a diagnostic potential for prostate cancer. PLoS One 2013;25:e70047.

19 Putz U, Howitt J, Doan A, Goh CP, Low LH, Silke J, Tan SS: The tumor suppressor PTEN is exported in exosomes and has phosphatase activity in recipient cells. Sci Signal 2012;5:ra70.

20 Putz U, Mah S, Goh CP, Low LH, Howitt J, Tan SS: PTEN secretion in exosomes. Methods 2015;77-78:157-163.

21 Kastl L, Brown I, Schofield AC: MiRNA-34a is associated with docetaxel resistance in human breast cancer cells. Breast Cancer Res Treat 2012;131:445-454.

22 Hu Q, Gong JP, Li J, Zhong SL, Chen WX, Zhang JY, Ma TF, Ji H, Lv MM, Zhao JH, Tang JH: Down-regulation of miRNA-452 is associated with adriamycin-resistance in breast cancer cells. Asian Pac J Cancer Prev 2014;15:5137-5142.

23 Hu Q, Chen WX, Zhong SL, Zhang JY, Ma TF, Ji H, Lv MM, Tang JH, Zhao JH: MicroRNA-452 contributes to the docetaxel resistance of breast cancer cells. Tumour Biol 2014;35:6327-6334.

24 Yang L, Li N, Wang H, Jia X, Wang X, Luo J: Altered microRNA expression in cisplatin-resistant ovarian cancer cells and upregulation of miR-130a associated with MDR1/P-glycoprotein-mediated drugresistance. Oncol Rep 2012;28:592-600.

25 Wang F, Li T, Zhang B, Li H, Wu Q Yang L, Nie Y, Wu K, Shi Y, Fan D: MicroRNA-19a/b regulates multidrug resistance in human gastric cancer cells by targeting PTEN. Biochem Biophys Res Commun 2013;434:688694. 